\title{
Self-adjustable offset min-sum algorithm for ISDB-S2 LDPC decoder
}

\author{
Wen Ji ${ }^{1 a)}$, Makoto Hamaminato ${ }^{1}$, Hiroshi Nakayama ${ }^{1}$, \\ and Satoshi Goto ${ }^{2}$ \\ ${ }^{1}$ Fujitsu Laboratories LTD. \\ 1-1, Kamikodanaka 4-chome, Nakahara-ku, Kawasaki, 211-8588, Japan \\ ${ }^{2}$ Graduate School of Information, Production \& Systems, Waseda University \\ 2-7 Hibikino, Wakamatsu-ku, Kitakyushu, Fukuoka, 808-0135, Japan \\ a) ji.wen@jp.fujitsu.com
}

\begin{abstract}
In this paper, a novel self-adjustable offset min-sum LDPC decoding algorithm is proposed for ISDB-S2 application. We present for the first time a uniform approximation of the check node operation through mathematical induction on Jacobian logarithm and theoretically shows that the offset value is mainly dependent on the difference of the two most unreliable inputs from the bit nodes, which makes the offset value adjustable during the iterative decoding procedure. Simulation results for all 11 code rates of ISDB-S2 demonstrate that the proposed method can achieve an average of $0.15 \mathrm{~dB}$ gain compared to the Min-sum based algorithms, and consumes only 1.21\% computation complexity compared to BP-based algorithms in the best case.
\end{abstract}

Keywords: LDPC decoding algorithm, ISDB-S2

Classification: Integrated circuits

\section{References}

[1] A. Hashimoto and Y. Suzuki, "A new transmission system for the advanced satellite broadcast," IEEE Trans. Consum. Electron., vol. 54, no. 2, pp. 353-360, May 2008.

[2] J. Chen, A. Dholakia, E. Eleftheriou, M. P. C. Fossorier, and X.-Y. Hu, "Reduced-complexity decoding of LDPC codes," IEEE Trans. Commun., vol. 53, no. 8, pp. 1288-1299, Aug. 2005.

[3] S. L. Howard, C. Schlegel, and V. C. Gaudet, "Degree-matched check node decoding for regular and irregular LDPCs," IEEE Trans. Circuits Syst. II, Exp. Briefs, vol. 53, no. 10, pp. 1054-1058, Oct. 2006.

[4] M. Jiang, C. Zhao, L. Zhang, and E. Xu, "Adaptive offset min-sum algorithm for low-density parity check codes," IEEE Commun. Lett., vol. 10, no. 6, pp. 483-485, June 2006.

[5] A. Anastasopoulos, "A comparison between the sum-product and the minsum iterative detection algorithms based on density evolution," IEEE Global Telecommun. Conf., vol. 2, pp. 1021-1025, Nov. 2001.

[6] L. Sakai, W. Matsumoto, and H. Yoshida, "Reduced complexity decoding based on approximation of update function for low-density parity-check 
codes," IEICE Trans. Fundamentals, vol. J90-A, no. 2, pp. 83-91, Feb. 2007.

[7] Y. Suzuki, A. Hashimoto, M. Kojima, S. Tanaka, T. Kimura, and T. Saito, "LDPC codes for the advanced digital satellite broadcasting system," IEICE Technical Report, vol. 109, no. 212, pp. 19-24, Sept. 2009.

\section{Introduction}

In Japan, a next generation satellite broadcasting system named ISDB-S2 (Integrated Services Digital Broadcasting via Satellite - Second Generation) was proposed by NHK (Japan Broadcasting Corporation) [1]. Low Density Parity Check (LDPC) code is selected as the error correction code to ensure the transmission quality and high error correction capability.

LDPC code can be efficiently decoded through Belief Propagation (BP) algorithm, though not hardware-friendly. Min-sum (MS) algorithm approximates BP algorithm with easy hardware implementation but greatly degrades the BER performance. Recently, two categories of schemes have been proposed to trade off between BER performance and hardware complexity: MS-based algorithms [2, 3, 4] and BP-based algorithms [5, 6]. Generally, BPbased algorithms outperform MS-based ones in BER performance with larger hardware cost. As high BER performance is in great demand for high-quality communication, and low hardware cost is in need for reducing manufacture cost, a decoding scheme which can achieve much higher BER performance than MS-based ones while drastically reducing the hardware cost of BP-based ones, is a must for practical ISDB-S2 application (Fig. 1).

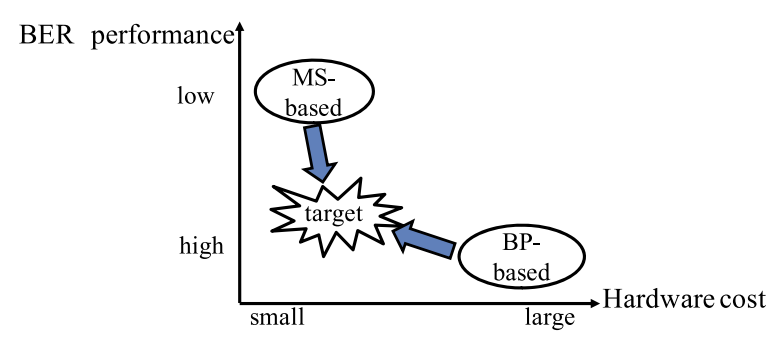

Fig. 1. Target for ISDB-S2 LDPC decoder

Motivated by this challenging design task, we proposed a hybrid decoding scheme as an initial attempt for both high BER performance and low hardware cost design. The algorithm improves the MS-based algorithm by a uniform approximation to the check node computation and the approximation is derived through mathematical induction on Jacobian logarithm used by BP-based algorithms. The simulation results further demonstrate that the proposed method can not only improve the BER performance compared to the MS-based algorithms with nearly no overhead in hardware cost, but also consumes far less hardware than the BP-based algorithms.

The rest of the paper is organized as follows. Section 2 describes LDPC 
decoding algorithms in detail. Section 3 discusses the proposed algorithm, its simulation result and hardware cost analysis, and finally Section 4 concludes.

\section{LDPC decoding algorithms}

Let $\alpha_{m n}$ be the message sent from check node $m$ to bit node $n, \beta_{m n}$ be the message sent from bit node $n$ to check node $m$, the check node operation of BP algorithm and MS algorithm can be expressed as Eq. (1) and Eq. (2). Note that $A(m)$ is defined as $A(m)=\left\{n \mid H_{m n}=1\right\}$.

$$
\begin{gathered}
\alpha_{m n}=2 \tanh ^{-1}\left(\prod_{n^{\prime} \in A(m) \backslash n} \tanh \left(\frac{\beta_{m n^{\prime}}}{2}\right)\right) \\
\alpha_{m n}=\prod_{n^{\prime} \in A(m) \backslash n} \operatorname{sign}\left(\beta_{m n^{\prime}}\right) \times \min _{n^{\prime} \in A(m) \backslash n}\left|\beta_{m n^{\prime}}\right|
\end{gathered}
$$

MS-based algorithms apply a normalization factor or offset factor to MS algorithm, i.e., Normalized Min-sum (NMS) algorithm and Offset Min-sum (OMS) algorithm [2]. Some recent progress claims that techniques to adjust the offset factor according to either the degree of the check node (DegreeMatched Min-sum (DMMS) algorithm [3]) or the smallest message sent from bit nodes (Adaptive Offset Min-sum (AOMS) algorithm [4]) can achieve better performance. However, DMMS requires significant computation power to determine the offset factor while AOMS needs Look Up Table (LUT) for implementation which increases the hardware cost.

BP-based algorithms, on the other hand, use the basic computation $\otimes$ (Eq. (3)) to implement Eq. (1) term by term, as shown in Eq. (4).

$$
\begin{gathered}
2 \tanh ^{-1}\left(\tanh \frac{\beta_{1}}{2} \times \tanh \frac{\beta_{2}}{2}\right)=\beta_{1} \otimes \beta_{2} \\
2 \tanh ^{-1}\left(\prod_{n^{\prime} \in A(m) \backslash n} \tanh \left(\frac{\beta_{m n^{\prime}}}{2}\right)\right)=\underbrace{\beta_{m 1} \otimes \beta_{m 2} \otimes \ldots \otimes \beta_{m n^{\prime}}}_{|A(m) \backslash n|}
\end{gathered}
$$

Eq. (3), the primitive form of Eq. (4), can be expanded using Jacobian $\operatorname{logarithm}\left(\ln \left(e^{a}+e^{b}\right)=\max (a, b)+\ln \left(1+e^{-|a-b|}\right)\right)$ twice as follows [5]:

$\beta_{1} \otimes \beta_{2}=\operatorname{sign}\left(\beta_{1}\right) \operatorname{sign}\left(\beta_{2}\right)\left(\left(\min \left(\left|\beta_{1}\right|,\left|\beta_{2}\right|\right)+f\left(\left|\beta_{1}\right|+\left|\beta_{2}\right|\right)-f\left(\left|\beta_{1}\right|-\left|\beta_{2}\right|\right)\right)\right.$ where $f(x)=\ln \left(1+e^{-|x|}\right)$

Since $\mathrm{f}(\mathrm{x})$ is not hardware friendly, Modified Min-sum (MMS) algorithm and Delta Min (DM) algorithm are proposed to approximate Eq. (5) $[5,6]$. Eq. (5) is then applied iteratively for the check node operation (Eq. (4)). Since altogether there are $|A(m)| \alpha$ values to be calculated in one row, the computation complexity of MMS and DM is proportional to $|A(m)| \times$ $(|A(m)|-2)$, which is relatively large for some codes in ISDB-S2. 


\section{Proposed algorithm}

\subsection{Proposed approximation of BP algorithm}

Since function $\otimes$ holds commutative law, we can fairly assume that $\left|\beta_{m 1}\right|<$ $\left|\beta_{m 2}\right|<\ldots<\left|\beta_{m n^{\prime}}\right|$. Thus a general case of Eq. (4) $\left(n^{\prime} \in A(m)\right.$ rather than $\left.n^{\prime} \in A(m) \backslash n\right)$ can be further expanded as Eq. (6) through a mathematical induction based on Eq. (5).

$$
\begin{aligned}
& \quad 2 \tanh ^{-1}\left(\tanh \left(\frac{\beta_{m 1}}{2}\right) \ldots \tanh \left(\frac{\beta_{m n^{\prime}}}{2}\right)\right) \\
& \approx \operatorname{sign}\left(\beta_{m 1}\right) \ldots \operatorname{sign}\left(\beta_{m n^{\prime}}\right)\left(\min \left(\left|\beta_{m 1}\right|, \ldots,\left|\beta_{m n^{\prime}}\right|\right)\right. \\
& \quad-f\left(\left|\beta_{m 2}\right|-\left|\beta_{m 1}\right|\right)-f\left(\left|\beta_{m 3}\right|-\left|\beta_{m 1}\right|\right)-\ldots-f\left(\left|\beta_{m n^{\prime}}\right|-\left|\beta_{m 1}\right|\right) \\
& \left.\quad+f\left(\left|\beta_{m 2}\right|+\left|\beta_{m 1}\right|\right)+f\left(\left|\beta_{m 3}\right|+\left|\beta_{m 1}\right|\right)+\ldots+f\left(\left|\beta_{m n^{\prime}}\right|+\left|\beta_{m 1}\right|\right)\right)
\end{aligned}
$$

The detailed proof of Eq. (6) is listed below.

(1) The condition of $n^{\prime}=2$ is already proved in Section 2.

(2) Suppose $n^{\prime}=k$ is correct, consider the situation of $n^{\prime}=k+1$

$$
\begin{aligned}
& \left.\begin{array}{l}
\tanh \left(\frac{\beta_{m 1}}{2}\right) \ldots \tanh \left(\frac{\beta_{m k}}{2}\right)=X \\
2 \tanh ^{-1}\left(\tanh \left(\frac{\beta_{m 1}}{2}\right) \ldots \tanh \left(\frac{\beta_{m k}}{2}\right)\right)=Y
\end{array}\right\} \Rightarrow X=\tanh \left(\frac{Y}{2}\right) \\
& 2 \tanh ^{-1}\left(\tanh \left(\frac{\beta_{m 1}}{2}\right) \ldots \tanh \left(\frac{\beta_{m k}}{2}\right) \tanh \left(\frac{\beta_{m(k+1)}}{2}\right)\right) \\
& =2 \tanh ^{-1}\left(\tanh \left(\frac{Y}{2}\right) \tanh \left(\frac{\beta_{m(k+1)}}{2}\right)\right) \\
& =\operatorname{sign}(Y) \operatorname{sign}\left(\beta_{m(k+1)}\right)\left(\min \left(|Y|,\left|\beta_{m(k+1)}\right|\right)\right. \\
& \left.+f\left(|Y|+\left|\beta_{m(k+1)}\right|\right)-f\left(\left|\beta_{m(k+1)}\right|-|Y|\right)\right) \\
& =\operatorname{sign}\left(\beta_{m 1}\right) \ldots \operatorname{sign}\left(\beta_{m k}\right) \operatorname{sign}\left(\beta_{m(k+1)}\right)\left(\operatorname { m i n } \left(\min \left(\left|\beta_{m 1}\right|, \ldots,\left|\beta_{m k}\right|\right)\right.\right. \\
& -f\left(\left|\beta_{m 2}\right|-\left|\beta_{m 1}\right|\right)-\ldots-f\left(\left|\beta_{m k}\right|-\left|\beta_{m 1}\right|\right) \\
& \left.+f\left(\left|\beta_{m 2}\right|+\left|\beta_{m 1}\right|\right)+\ldots+f\left(\left|\beta_{m k}\right|+\left|\beta_{m 1}\right|\right),\left|\beta_{m(k+1)}\right|\right) \\
& \left.-f\left(\left|\beta_{m(k+1)}\right|-|Y|\right)+f\left(\left|\beta_{m(k+1)}\right|+|Y|\right)\right) \\
& \because \min \left(\left|\beta_{m 1}\right|, \ldots,\left|\beta_{m k}\right|\right)=\min \left(\left|\beta_{m 1}\right|, \ldots,\left|\beta_{m k}\right|,\left|\beta_{m(k+1)}\right|\right)=\left|\beta_{m 1}\right| \\
& |Y| \approx \min \left(\left|\beta_{m 1}\right|, \ldots,\left|\beta_{m k}\right|\right)=\left|\beta_{m 1}\right| \\
& \therefore 2 \tanh ^{-1}\left(\tanh \left(\frac{\beta_{m 1}}{2}\right) \ldots \tanh \left(\frac{\beta_{m k}}{2}\right) \tanh \left(\frac{\beta_{m(k+1)}}{2}\right)\right) \\
& \approx \operatorname{sign}\left(\beta_{m 1}\right) \ldots \operatorname{sign}\left(\beta_{m(k+1)}\right)\left(\min \left(\left|\beta_{m 1}\right|, \ldots,\left|\beta_{m k}\right|,\left|\beta_{m(k+1)}\right|\right)\right. \\
& -f\left(\left|\beta_{m 2}\right|-\left|\beta_{m 1}\right|\right)-f\left(\left|\beta_{m 3}\right|-\left|\beta_{m 1}\right|\right)-\ldots-f\left(\left|\beta_{m(k+1)}\right|-\left|\beta_{m 1}\right|\right) \\
& \left.+f\left(\left|\beta_{m 2}\right|+\left|\beta_{m 1}\right|\right)+f\left(\left|\beta_{m 3}\right|+\left|\beta_{m 1}\right|\right)+\ldots+f\left(\left|\beta_{m(k+1)}\right|+\left|\beta_{m 1}\right|\right)\right)
\end{aligned}
$$

Since $|A(m)|$ is usually a large number for ISDB-S2, the implementation of Eq. (6) requires a large amount of hardware resources. Hence an efficient approximation is a necessity. As $\mathrm{f}(\mathrm{x})$ is a monotonically decreasing function with $f(x) \doteq 0$ when $x>2.5$, and $\left|\beta_{m 1}\right|, \ldots,\left|\beta_{m n^{\prime}}\right|$ have the monotonically 
increasing relationship, we can derive that $\left|\beta_{m 2}\right|-\left|\beta_{m 1}\right|$ is the smallest one among all the arguments of $f(x)$, thus $-f\left(\left|\beta_{m 2}\right|-\left|\beta_{m 1}\right|\right)$ becomes the dominant $f(x)$ term. By approximating all the other terms through multiplying a normalization factor $\gamma$ to the dominant one (we use $-f\left(\left|\beta_{\min 2}\right|-\left|\beta_{\min 1}\right|\right.$ ) from now on), we finally obtain Eq. (7) as the approximation to Eq. (1). Note that, here $n^{\prime} \in A(m) \backslash n$ in Eq. (4) rather than $n^{\prime} \in A(m)$ is considered, and for those two cases when the excluded item $\beta_{m n}$ happens to be either $\beta_{\min 1}$ or $\beta_{\min 2}$, we use the same $-f\left(\left|\beta_{\min 1}\right|-\left|\beta_{\min 2}\right|\right)$ value.

$$
\alpha_{m n} \approx \prod_{n^{\prime} \in A(m) \backslash n} \operatorname{sign}\left(\beta_{m n^{\prime}}\right)\left(\min _{n^{\prime} \in A(m) \backslash n}\left|\beta_{m n^{\prime}}\right|-\gamma f\left(\left|\beta_{m i n 2}\right|-\left|\beta_{m i n 1}\right|\right)\right)
$$

From Eq. (7), we can see that the offset factor is self-adjustable, during the iterative decoding, according to the difference of the two most unreliable inputs from the bit nodes. Such adjustable scheme precisely models the variations of bit node messages, hence enhances the decoding efficiency.

\subsection{Simulation results}

Software simulation of BP, NMS, OMS, DMMS, AOMS, MMS, DM and the proposed decoding algorithm using layered schedule has been conducted for all 11 parity check matrices in ISDB-S2. Except BP algorithm is simulated using floating values, all intermediate messages of simulations for the other algorithms are coded in 6 bit sign-magnitude format. For simple hardware implementation, we use $\Delta$ function $\Delta(x)=\max \left(\frac{5}{8}-\frac{|x|}{4}, 0\right)$ for approximation of function $f(x)$ in Eq. (7). Also, the QPSK modulation and AWGN channel is modeled, and the maximum number of iteration is set to 50 .

Fig. 2 (a) illustrates the BER performance for rate $\frac{3}{4}$, which will be mainly used in ISDB-S2 service. It can be observed from the figure that the proposed algorithm achieves at least $0.2 \mathrm{~dB}$ gain compared to the MS-based algorithms, and sometimes even outperforms BP-based algorithms.

To further evaluate the BER performance of the proposed algorithm and its suitability to all the ISDB-S2 LDPC codes, we use the required CNR as a metric defined as the carrier-to-noise ratio when the BER exceeds $10^{-11}$, and

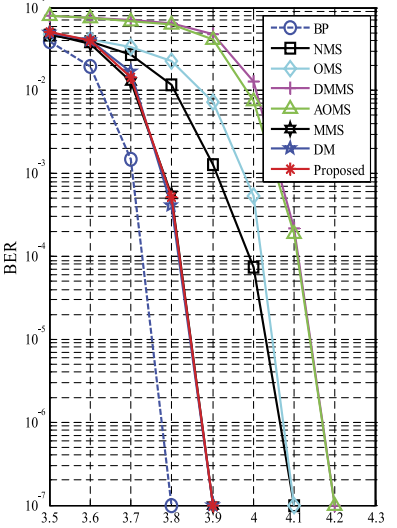

(a) BER performance for rate $3 / 4$

\begin{tabular}{|c|c|c|c|c|c|c|c|c|}
\hline \multirow{2}{*}{ Rate } & \multirow{2}{*}{$\begin{array}{c}\text { BP } \\
\text { floating } \\
\text { point) }\end{array}$} & \multicolumn{9}{|c|}{ MS-based } & \multicolumn{2}{|c|}{ BP-based } & \multirow{2}{*}{ Proposed } \\
\cline { 3 - 8 } & NMS & OMS & DMMS & AOMS & MMS & DM & \\
\hline \hline $1 / 4$ & -2.15 & -1.56 & -1.33 & -1.49 & -1.52 & -1.73 & -1.56 & -1.55 \\
\hline $1 / 3$ & -1.14 & -0.60 & -0.45 & -0.42 & -0.41 & -0.94 & -0.86 & -0.67 \\
\hline $2 / 5$ & -0.16 & 0.31 & 0.36 & 0.44 & 0.54 & 0.04 & 0.13 & 0.34 \\
\hline $1 / 2$ & 1.05 & 1.42 & 1.46 & 1.45 & 1.44 & 1.14 & 1.24 & 1.38 \\
\hline $3 / 5$ & 2.35 & 2.67 & 2.67 & 2.55 & 2.54 & 2.46 & 2.45 & 2.45 \\
\hline $2 / 3$ & 3.27 & 3.47 & 3.46 & 3.34 & 3.34 & 3.26 & 3.25 & 3.25 \\
\hline $3 / 4$ & 3.95 & 4.29 & 4.26 & 4.36 & 4.36 & 4.06 & 4.05 & 4.05 \\
\hline $4 / 5$ & 4.97 & 5.28 & 5.37 & 5.15 & 5.16 & 5.08 & 4.95 & 5.07 \\
\hline $5 / 6$ & 5.48 & 5.79 & 6.01 & 5.66 & 5.66 & 5.46 & 5.46 & 5.45 \\
\hline $7 / 8$ & 5.97 & 6.16 & 6.08 & 6.15 & 6.15 & 6.01 & 5.96 & 6.07 \\
\hline $9 / 10$ & 6.79 & 7.09 & 6.89 & 6.87 & 6.87 & 6.78 & 6.78 & 6.88 \\
\hline \hline Average & 2.76 & 3.12 & 3.16 & 3.1 & 3.1 & 2.87 & 2.9 & 2.97 \\
\hline
\end{tabular}

(b) Required CNR (dB)

Fig. 2. Performance evaluation 
the same evaluation method, namely extrapolation, to calculate the required CNR as in [7]. The results are listed in Fig. 2 (b). The row Average indicates the average required $\mathrm{CNR}$ for all the 11 codes. We can see that the required CNR of the proposed algorithm $(2.97 \mathrm{~dB})$ is about $0.15 \mathrm{~dB}$ better than the MS-based ones in average $((3.12+3.16+3.1+3.1) / 4=3.12 \mathrm{~dB})$.

\subsection{Comparison of computation complexity and hardware cost}

Although the average required CNR of BP-based algorithms is $0.09 \mathrm{~dB}$ better than the proposed algorithm, their computation complexity and hardware cost become the obstacle of practical application and cannot be neglected. The comparison of computation complexity and the hardware cost of the check node operation for one row (exclude the sign computation) are listed in Fig. 3 (a). Under column Computation Complexity, [comp], $[a d d]$, $[$ shift $]$ indicate the computation complexity of comparison operation, addition or subtraction operation, and shift operation, respectively. Since each operation takes one clock cycle, the computation complexity of $[c o m p]:[a d d]:[$ shift $]$ should be 1:1:1. According to Fig. $3(\mathrm{a})$, for rate $\frac{9}{10}$ with the largest row weight $|A(m)|$ of 32 , the computation complexity relation between NMS, OMS, DMMS, AOMS, MMS, DM and the proposed method is $1.03: 1.03: 1.22: 1.05: 90: 75: 1.09$. We can see that the proposed algorithm consumes only $1.21 \%$ (1.09/90) computation complexity of MMS. The estimation results of hardware cost are also listed under column Hardware Cost. [adder 5$]$ and [adder6] indicate the cost of an adder or subtractor for 5 bits and 6 bits. Note that we assume a comparator shares a similar cost with an adder, and we neglect the cost for shifter. In order to apply the same

\begin{tabular}{|c|c|c|c|}
\hline \multirow{2}{*}{ Computation Complexity } & \multicolumn{2}{c|}{ Hardware Cost } \\
\cline { 3 - 4 } & $2 \times|\mathrm{A}(\mathrm{m})| \times[$ comp $]+2 \times[$ shift $]$ & Number of Computation Units & Gate Count \\
\hline \hline NMS & $2 \times|\mathrm{A}(\mathrm{m})| \times[$ comp $]+2 \times[$ add $]$ & $2 \times[$ adder 5$]$ & 60 \\
\hline OMS & $(2 \times|\mathrm{A}(\mathrm{m})|+4) \times[$ comp $]+3 \times[$ shift $]+7 \times[$ add $]$ & $5 \times[$ adder 5$]+6 \times[$ adder 6$]$ & 366 \\
\hline DMMS & $2 \times|\mathrm{A}(\mathrm{m})| \times[$ comp $]+1 \times[$ shift $]+2 \times[$ add $]$ & $4 \times[$ adder 5$]+8 w o r d \times 3$ bit $[$ LUT $]$ & 360 \\
\hline AOMS & $|\mathrm{A}(\mathrm{m})| \times(|\mathrm{A}(\mathrm{m})|-2) \times(3 \times[$ comp $]+3 \times[$ add $])$ & $|\mathrm{A}(\mathrm{m})| \times(4 \times[$ adder 6$]+2 \times[$ adder 5$])$ & 6528 \\
\hline MMS & $(2 \times|\mathrm{A}(\mathrm{m})|) \times[$ comp $]+4 \times[$ add $]+2 \times[$ shift $]$ & $|\mathrm{A}(\mathrm{m})| \times(4 \times[$ adder 5$])$ & 3840 \\
\hline DM & $|\mathrm{A}(\mathrm{m})| \times(|\mathrm{A}(\mathrm{m})|-2) \times(1 \times[$ comp $]+3 \times[$ add $]+1 \times[$ shift $])$ & $6 \times[$ adder 5$]$ & 180 \\
\hline proposed & &
\end{tabular}

(a) Computation complexity and hardware cost

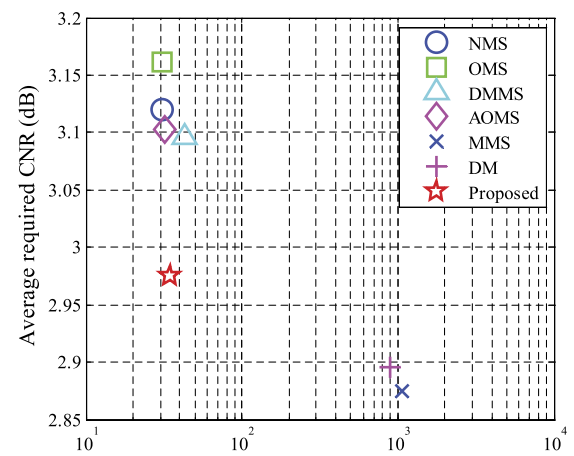

(b) Average computation complexity (times)

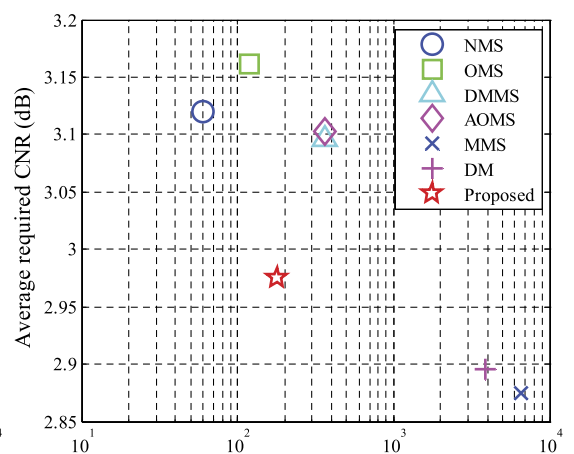

(c) Area (gates)

Fig. 3. Computation complexity and hardware cost 
design to all the rates of LDPC code rates in ISDB-S2, $|A(m)|$ is chosen as the largest one which is 32 for rate $\frac{9}{10}$. The gate count is estimated with the adder as 6 gates per bit and the LUT as 10 gates per bit. The results are listed under column Gate Count. We can see that the proportion of the average area of MS-based algorithm, the area of the proposed algorithm and the average area of BP-based algorithm is $(60+120+366+360) / 4: 180$ : $(6528+3840) / 2=4.4: 3.5: 100$, which indicates that the proposed algorithm not only consumes $3.5 \%$ hardware cost compared to BP-based algorithms in average but also consumes less hardware than some of the MS-based algorithms.

Fig. 3 (b) shows the relation of average computation complexity and average required CNR and Fig. 3 (c) shows the relation of area cost and average required CNR for all the rates in ISDB-S2. We can figure out from the two figures that averagely the proposed algorithm can achieve $0.15 \mathrm{~dB}$ BER performance gain with almost the same area as MS-based ones, which meets the target in Fig. 1. This satisfactory result that the total performance of the proposed algorithm is better than MS-based algorithm and BP-based algorithm, makes it an excellent candidate for ISDB-S2 decoder application.

\section{Conclusion}

In this paper, a novel self-adjustable offset min-sum algorithm is proposed with the check node operation approximating BP algorithm using Jacobian logarithm iteratively. The proposed algorithm is hardware-friendly compared to the BP-based algorithms and can achieve an average of $0.15 \mathrm{~dB}$ gain compared to Min-sum based algorithms for ISDB-S2 LDPC codes. 